\title{
Assessing HIV-Related Stigma in Healthcare Settings in the Era of the COVID-19 Pandemic, Pittsburgh, Pennsylvania
}

\author{
Sarah Krier ${ }^{1} \cdot$ Corrine Bozich ${ }^{1} \cdot$ Robert Pompa $^{2} \cdot$ M. Reuel Friedman ${ }^{1}$
}

Published online: 2 May 2020

(c) Springer Science+Business Media, LLC, part of Springer Nature 2020

Reducing stigma and eliminating discrimination associated with HIV status is a key strategy in Pennsylvania's Integrated HIV Prevention and Care Plan. In collaboration with the Pennsylvania HIV Planning Group (HPG) we developed an intersectional stigma measure to examine experiences of stigma and discrimination in healthcare settings among individuals living with HIV (PLWH). This survey is ongoing and aims to assess the prevalence of multidimensional stigma in healthcare settings, characterize intersectional stigma burden, and evaluate the impact of stigma on health among a sample of individuals living with HIV in Pennsylvania who are recipients of the AIDS Drug Assistance Program. In this Note from the Field, we describe the unprompted COVID-related responses we have received since March 4, 2020 until the writing of this Note (April 14, 2020). These responses present an important opportunity to begin to learn about how the COVID-19 pandemic is affecting the lives of people living with HIV and their beliefs and attitudes about their healthcare needs and experiences.

In our research we asked participants to respond to three open-text questions: (1) "What are some concerns you may have about your future healthcare needs and options?"; (2) "What are you most worried about in your next healthcarerelated experience?"; and (3) "What are you most looking forward to in your next healthcare experience?" Qualitative analysis of recorded data used inductive grounded theory approaches [1, 2]. Between March 4 and April 14, 2020, we received 19 COVID-19 related responses to the opentext questions from 16 participants (Table 1). Of the 16 participants, 94\% identified as male; $94 \%$ identified as White; and $75 \%$ identified as gay. The age of participants ranged

Sarah Krier

SEK29@pitt.edu

1 Department of Infectious Diseases and Microbiology, Graduate School of Public Health, University of Pittsburgh, 3520 Fifth Avenue, Suite 400, Pittsburgh, PA 15213, USA

2 Jim Thorpe, PA, USA from 29 to 72, with a mean age of 58.7. Participants represented four Ryan White regional grantee regions of the seven regions in the Commonwealth. This is a subsample of a larger, more diverse sample of respondents.

Many participants expressed concern and worry about COVID-19 with references to "the new virus." As one participant explained: "Honestly? In plain English, I am scared shitless of the corona virus." Concerns and worry related to living with HIV in the era of the COVID-19 pandemic was a key theme in the COVID-19 related responses. When describing future healthcare concerns, needs and options, one participant offered: "Now? Having HIV and coronavirus. Scary..." Another participant said: "I am hoping I don't need service soon with the virus thing going around and being with a compromised immune system." The possible negative impact of COVID-19 on the navigation of HIV and other healthcare needs was also a concern:

I'm terrified that my needs and options will become harder to have due to the long-lasting time that I'm hearing that the Coronavirus will be lasting, i.e., resources will be fewer and more difficult to find. Also, I am on salvage therapy and my options to treatments are fewer and my hearing bad news becomes more. I feel like a deer facing headlights.

Participants expressed fears of how the COVID-19 pandemic will disrupt HIV care services, like the loss of, or restricted access to healthcare services and HIV funding. In addition, the impacts of the current social distancing mandates on mental health and wellbeing is concerning: "Getting by this corona virus period of virtual isolation. Normally, I go the gym twice daily and eat out an awful lot. I, like all, am concerned about how long this situation will continue."

Another common healthcare concern among participants was in navigating cancer care in the era of the COVID19 pandemic. Participants noted concerns for addressing multiple skin cancers sites, prostate cancer, and "cancer returning." As one participant notes: "Everything being 
Table 1 Responses Related to COVID-19

What are you most worried about in your next healthcare-related experience?

What are you most looking forward to in your next healthcare-related experience?

What are some concerns you may have about your future healthcare needs and options?

\section{Coronavirus}

Prostate cancer, corona virus (COVID-19)

The coronavirus

I am hoping I don't need service soon with the virus thing going around and being with a compromised immune system

Everything being complicated with fear and anxiety associated with having my condition during the Coronavirus period, safely arriving and exiting to my appointment in order to have to repeat a biopsy is what I'm worried about

This survey is being answered during the height of the corona virus restrictions

Multiple skin cancer sites, coronavirus

coronavirus

Covid-19 and cancer returning

The Crona 19 virus

Ninety Nine percent of My health-care experiences are great so I'm not expect anything more than that... I changed HIV doctors and due to the coronavirus I had an appointment over the phone... it was good

My next appointment (hopefully) is my PCP/Infectious disease physician. He is fantastic as my doctor and very close as a friend. He is also recognized for his intelligence broadcasting on coronavirus

Telemedicine visit possibly replace my appointment CORONAVIRUS!!!!!!!!!!!!!!

I'm terrified that my needs and options will become harder to have due to the long lasting time that I' $m$ hearing that the Coronavirus will be lasting, i.e., resources will be fewer and more difficult to find. Also, I am on salvage therapy and my options to treatments are fewer and my hearing bad news becomes more. I feel like a deer facing headlights

Getting by this coronavirus period of virtual isolation. Normally, I go the gym twice daily and eat out an awful lot. I, like all, am concerned about how long this situation will continue

Honestly? In plain English, I am scared shitless of the coronavirus

Now? Having HIV and coronavirus. Scary...

that virus going around complicated with fear and anxiety associated with having my condition during the Coronavirus period, safely arriving and exiting to my appointment in order to have to repeat a biopsy is what I'm worried about."

While the majority of COVID-19 responses expressed concern and worry, one participant explained: "I changed HIV doctors and due to the coronavirus I had an appointment over the phone... it was good." One participant also mentioned how he was looking forward to the possible introduction of telemedicine for this next healthcare appointment: "Telemedicine visit possibly replace my appointment." Another respondent expressed their appreciation and respect for their infectious disease physician and their expertise with COVID-19: "My next appointment (hopefully) is my primary care/Infectious disease physician. He is fantastic as my doctor and very close as a friend. He is also recognized for his intelligence broadcasting his information of coronavirus."
These responses emerged unprompted, utilizing opentext boxes from an online survey currently being deployed in the field. The COVID-19 related responses that we have received to date indicate the great concern that PLWH feel about potential dual diagnoses with COVID-19 and increased susceptibility to poor COVID outcomes as a result of HIV positive status. These unprompted responses covered several domains of concern including avoidance of healthcare services due to nosocomial risk of COVID infection, social isolation, change in routine, potential mortality, continued access to healthcare services, and management of comorbidities, specifically cancer. In addition, the utilization of telemedicine for healthcare and trust in practitioner's advice during the COVID-19 pandemic emerged as positive domains.

Emerging research is showing that people with HIV should be a considered a high-risk population for COVID19 because of the prevalence of: older individuals living 
with HIV and the devastating impact COVID-19 is having on older people; potential immunological challenges, and; a high prevalence of comorbidities like diabetes, cancer, hypertension, mental health and substance use, conditions which are implicated in poorer COVID-19 outcomes [3, 4]. This ongoing survey strives to assess the prevalence of internalized, anticipated and enacted intersectional stigma in healthcare settings to support a better understanding of how stigma impacts health and well-being and to inform the development of interventions to reduce stigma in healthcare settings and improve HIV prevention and care outcomes in Pennsylvania. We did not consider the specific challenges that have arisen for PLWH who are now at heightened risk as a result of the COVID-19 pandemic.

These survey responses allow us to better understand the barriers that PLWH may face during the COVID-19 pandemic and beyond. PLWH are navigating their HIV and related healthcare needs in the era of the COVID-19 pandemic, in addition to navigating COVID-19 prevention and care while living with HIV. Living with a stigmatized chronic infectious disease in the times of a pandemic presents a myriad of challenges. This is particularly true in communities of color and SGM communities who experience systemic social and health inequities [5, 6]. Gross disparities in our society around race, sexual, gender identities, employment, health, and education lead many populations to experience discrimination, unwelcome attitudes, and lack of understanding from healthcare staff [4]. COVID-19 is exacerbating these inequities in terms of housing stability, employment status, healthcare access, and food security. This is further demonstrated in a recent analysis published by the CDC that shows African American are disproportionately in need of COVID-related hospital care [6].

In addition, we expect that the historic and pervasive effects of HIV-related stigma are likely hitting close to home for many individuals living with HIV in the era of the COVID-19 pandemic. As PLWH self-isolate to prevent COVID-19, we need to recognize that enforced social isolation in populations who already report stigma, alienation and discrimination may exacerbate underlying psychosocial health conditions. Studies have shown that people living with HIV are already more likely to experience loneliness due to a history of stigma and discrimination, with studies also indicating that higher levels of loneliness and social isolation result in greater negative health outcomes, including a higher likelihood of smoking, at-risk drinking or substance use, higher rates or depression, and lower quality of life [7]. We need work to understand the effects of social isolation on individuals living with HIV, how they are seeking support and how they want to be supported. It is now even more important to work on short-term interventions to increase social connectedness during these times of isolation.
Moving forward, we must try to assess the impact of COVID-19 on the health and well-being of people living with and at risk for HIV. How has this pandemic impacted medical adherence, healthcare engagement and mental health? Will the COVID-19 pandemic be associated with higher rates of internalized and perceived stigma, as well as lower rates of medical adherence, lower health engagement, and poorer mental health among individuals living with HIV? Will the utilization of telemedicine for HIV care services increase the capacity and support in the reduction of stigma and discrimination that PLWH face in community and healthcare settings? Over time, we will see the effects that COVID-19 has on individuals living with HIV's engagement in healthcare. These unprompted responses about COVID-19 offer important preliminary insights into how the COVID-19 pandemic is affecting the lives of people living with HIV and their beliefs and attitudes about their healthcare needs and experiences in the future.

\section{References}

1. Foley G, Timonen V. Using grounded theory method to capture and analyze health care experiences. Health Serv. Res. 2014;50(4):1195-210.

2. Ulin PR, Robinson ET, Tolley EE. Qualitative methods in public health: a field guide for applied research. Jossey-Bass: Wiley; 2005 .

3. Interim Guidance for COVID-19 and Persons with HIV. National Institutes of Health: U.S. Department of Health and Human Services; [updated 2020 Mar 20; cited 2020 Apr 08]. https://aidsi nfo.nih.gov/guidelines/html/8/covid-19-and-persons-with-hiv-interim-guidance-/0?fbclid=IwAR3KY84zf_z5BoBfeV1xB8Srg NzMR8M96bAf0f7qjzVhGsuKOEjlA-MGC4A.

4. Open Letter About Coronavirus and the LGBTQ Communities. GLMA: GLMA; [cited 2020 Apr 08]. www.glma.org/index .cfm? fuseaction $=$ Feature . showFeature $\&$ CategoryID $=1 \&$ Featu reID $=853$

5. Top physician orgs urge COVID-19 mortality data by race, ethnicity. American Medical Association: American Medical Association; [updated Apr 8, 2020; cited 2020 Apr 13]. www.ama-assn. org/press-center/press-releases/top-physician-orgs-urge-covid -19-mortality-data-race-ethnicity.

6. Garg S, Kim L, Whitaker M. Hospitalization Rates and Characteristics of Patients Hospitalized with Laboratory-Confirmed Coronavirus Disease 2019_COVID-NET, 14 States, March 1-30, 2020. Centers for Disease Control and Prevention: Centers for Disease Control and Prevention; 2020 [updated 2020 Apr 8; cited 2020 Apr 10]. https://www.cdc.gov/mmwr/volumes/69/wr/mm691 5e3.htm?s_cid=mm6915e3_w.

7. Greene M, Hessol NA, Perissinotto C, Zepf R, Hutton Parrott A, Foreman C, et al. Loneliness in older adults living with HIV. AIDS Behav. 2018;22(5):1475-84.

Publisher's Note Springer Nature remains neutral with regard to jurisdictional claims in published maps and institutional affiliations. 